\title{
STRONG CONVERGENCE THEOREM FOR FINITE FAMILY OF GENERALISED ASYMPTOTICALLY NONEXPANSIVE MAPS
}

\author{
AGATHA CHIZOBA NNUBIA ${ }^{1}$, SHEILA AMINA BISHOP ${ }^{2, *}$ \\ ${ }^{1}$ Department of Mathematics, Nnamdi Azikiwe University, Awka, Nigeria \\ ${ }^{2}$ Department of Mathematics, Convenant University, Ota, Nigeria \\ ${ }^{*}$ Corresponding author: sheilabishop95@yahoo.com; sheila.bishop@covenantuniersity.edu.ng \\ Abstract. Let $K$ be a nonexpansive retract of a uniformly convex Banach space $X$ with retraction $P$ and \\ $T_{i: 1 \cdots, m}: K \longrightarrow X$ a finite family of uniformly continuous generalised asymptotically nonexpansive maps \\ with a nonempty common fixed point set $F$. We provided and proved sufficient conditions for the strong \\ convergence of a sequence of successive approximations generated by an m-step algorithm to a point of $F$.
}

\section{INTRODUCTION}

Let $K$ be a nonempty subset of a normed linear space $E . K$ is said to be (sequentially) compact if every closed bounded sequence in $K$ has a subsequence that converges in $K . K$ is said to be boundedly compact if every bounded subset of $K$ is compact. Finite dimensional spaces are boundedly compact. Given a subset $S$ of $K$, we shall denote by $\operatorname{co}(S)$ and $\operatorname{ccl}(S)$ the convex hull and the closed convex hull of $S$ respectively. If $K$ is boundedly compact convex and $S$ is bounded, then $\operatorname{co}(S)$ and hence $\operatorname{ccl}(S)$ are compact convex subsets of $K$.

A map $T: K \rightarrow E$ is said to be semi-compact if for any bounded sequence $\left\{x_{n}\right\} \subset K$ such that

Received 2019-06-14; accepted 2019-07-18; published 2020-01-02.

2010 Mathematics Subject Classification. 47H09, 47H10.

Key words and phrases. common fixed point set; generalised asymptotically nonexpansive maps; strong convergence; uniformly convex Banach space.

(C)2020 Authors retain the copyrights of their papers, and all open access articles are distributed under the terms of the Creative Commons Attribution License. 
$\left\|x_{n}-T x_{n}\right\| \rightarrow 0$ as $n \rightarrow \infty$ there exists a subsequence $\left\{x_{n_{j}}\right\}$ of $\left\{x_{n}\right\}$ such that $x_{n_{j}}$ converges strongly to some $x^{*} \in K$ as $j \rightarrow \infty$. The map $T$ is said to be demi-compact at $z \in E$ if for any bounded sequence $\left\{x_{n}\right\} \subset K$ such that $\left\|x_{n}-T x_{n}\right\| \rightarrow z$ as $n \rightarrow \infty$ there exist a subsequence $\left\{x_{n_{j}}\right\}$ of $\left\{x_{n}\right\}$ and a point $p \in K$ such that $x_{n_{j}}$ converges strongly to $p$ as $j \rightarrow \infty$. (Observe that if $T$ is additionally continuous, then $p-T p=z)$. A nonlinear map $T: K \rightarrow E$ is said to be completely continuous if it maps bounded sets into relatively compact sets. A mapping $T: K \longrightarrow E$ is called nonexpansive if and only if for all $x, y \in K$, we have that

$$
\|T x-T y\| \leq\|x-y\|
$$

$K$ is called a nonexpansive retract of $E$ if there exists a nonexpansive map $P: E \longrightarrow K$ which is onto and such that $P^{2}=I$. The map $P$ is called the nonexpansive retraction of $E$ onto $K$. Let $K$ be a nonempty subset of a real normed space $E$. Let $P: E \longrightarrow K$ be a nonexpansive retraction of $E$ onto $K$. A nonself map $T: K \longrightarrow E$ is called asymptotically nonexpansive mapping if and only if there exists a sequence $\left\{\mu_{n}\right\}_{n \geq 1} \subset[0,+\infty)$, with $\lim _{n \rightarrow \infty} \mu_{n}=0$ such that for all $x, y \in K$,

$$
\left\|T\left(P_{K} T\right)^{n-1} x-T\left(P_{K} T\right)^{n-1} y\right\| \leq\left(1+\mu_{n}\right)\|x-y\| \quad \forall n \in \mathbb{N}
$$

where $P_{K}: X \longrightarrow K$ is nonexpansive retraction of $E$ onto $K . T$ is called generalised asymptotically nonexpansive mapping if and only if there exist a sequences $\left\{\mu_{n}\right\}_{n \geq 1},\left\{\eta_{n}\right\}_{n \geq 1} \subset[0,+\infty)$, with $\lim _{n \rightarrow \infty} \mu_{n}=1$ and $\lim _{n \rightarrow \infty} \eta_{n}=0$ such that for all $x, y \in D(T)$,

$$
\left\|T\left(P_{K} T\right)^{n-1} x-T\left(P_{K} T\right)^{n-1} y\right\| \leq \mu_{n}\|x-y\|+\eta_{n} \quad n \geq 1
$$

Goebel and Kirk [3] introduced the class of asymptotically nonexpansive mappings as a generalisation of nonexpansive mappings, Zegeye and Shahzad [13] introduced the class of generalised asymptotically nonexpansive mappings as a generalization of asymptotically nonexpansive maps. As further generalisation, Alber, Chidume and Zegeye [1] introduced the class of total asymptotically nonexpansive mappings, where $T: K \longrightarrow H$ is called total asymptotically nonexpansive if and only if there exist two sequences $\left\{\mu_{n}\right\}_{n \geq 1},\left\{\eta_{n}\right\}_{n \geq 1} \subset[0,+\infty)$, with $\lim _{n \rightarrow \infty} \mu_{n}=0=\lim _{n \rightarrow \infty} \eta_{n}$ and nondecreasing continuous function $\phi:[0,+\infty) \longrightarrow[0,+\infty)$ with $\phi(0)=0$ such that for all $x, y \in K$,

$$
\left\|T\left(P_{K} T\right)^{n-1} x-T\left(P_{K} T\right)^{n-1} y\right\| \leq\|x-y\|+\mu_{n} \phi(\|x-y\|)+\eta_{n} \quad n \geq 1 .
$$

Ofoedu and Nnubia [8] gave an example to show that the class of asymptotically nonexpansive mappings is a proper subset of the class of total asymptotically nonexpansive mappings. The class of total asymptotically nonexpansive type mappings includes the class of mappings which are asymptotically nonexpansive in the intermediate sense. These classes of mappings had been studied by several authors (see e.g., [3], [5], [11], [14]). 


\section{Preliminaries}

We shall make use of the following result:

A Banach space $E$ is said to satisfy Opial's condition if for each sequence $\left\{x_{n}\right\}_{n \geq 1} \in E$ which converges weakly to a point $z \in E$, we have that $\liminf _{n \rightarrow \infty}\left\|x_{n}-z\right\|<\liminf _{n \rightarrow \infty}\left\|x_{n}-y\right\|, \forall y \in E$, such that $y \neq z$. It is well known that every Hilbert space satisfies Opial's condition(see e.g., [9]).

A map $\mathrm{T}$ is said to satisfy condition $B$ if there exists $f:[0, \infty) \rightarrow[0, \infty)$ strictly increasing, continuous, $f(0)=0$ such that for all $x \in D(T),\|x-T x\| \geq f(d(x, F))$ where $F=F(T)=\{x \in D(T): x=T x\}$ and $d(x, F)=\inf \{\|x-y\|: y \in F\}$.

Lemma 2.1. [2] Let $H$ be a real Hilbert space. Then for all $x, y \in H$ the following inequality holds.

$$
\|x+y\|^{2} \leq\|x\|^{2}+2\langle y, x+y\rangle
$$

Lemma 2.2. [2] For any $x, y, z$ in a real Hilbert space $H$ and a real number $\lambda \in[0,1]$,

$$
\|\lambda x+(1-\lambda) y-z\|^{2}=\lambda\|x-z\|^{2}+(1-\lambda)\|y-z\|^{2}-\lambda(1-\lambda)\|x-y\|^{2} .
$$

Lemma 2.3. [12] Let $\left\{x_{n}\right\}$ be sequence of nonegative real numbers satisfying the following relation:

$$
x_{n+1} \leq x_{n}-\alpha_{n} x_{n}+\delta_{n}, \quad n \geq n_{0},
$$

where $\left\{\alpha_{n}\right\}_{n \geq 1} \subset(0,1)$ and $\left\{\delta_{n}\right\}_{n \geq 1} \subset \mathbb{R}$ satisfying the following conditions:

$\sum_{n=0}^{\infty} \alpha_{n}=\infty, \lim _{n \rightarrow \infty} \alpha_{n}=0$ and $\limsup _{n \rightarrow \infty} \delta_{n} \leq 0$, then $\lim _{n \rightarrow \infty} x_{n}=0$.

Lemma 2.4. Let $\left\{\mu_{n}\right\},\left\{\nu_{n}\right\}$ and $\left\{\eta_{n}\right\}$ be nonnegative sequences such that $\sum_{n \geq 0} \nu_{n}<\infty, \sum_{n \geq 0} \eta_{n}<\infty$ and $\mu_{n+1} \leq\left(1+\nu_{n}\right) \mu_{n}+\eta_{n}$. Then $\lim _{n \rightarrow \infty} \mu_{n}$ exists.

The nearest point projection $P_{K}: H \longrightarrow K$ defined from $H$ onto $K$ is the function which assign to each $x \in H$ its nearest point denoted by $P_{K} x \in K$. Thus $P_{K} x$ is the unique point in $K$ such that $\left\|x-P_{K} x\right\| \leq\|x-y\|$ for all $y \in K$ and we have the following Lemmas.

Lemma 2.5. [12]. Let $K$ be a closed convex nonempty subset of a real Hilbert space $H$. Let $x \in H$, then $z=P_{K} x$ if and only if

$$
\langle x-z, y-z\rangle \leq 0 \forall y \in K
$$

Lemma 2.6. Let $K$ be a closed convex nonempty subset of a Banach space $E$ and let $T_{i \in I}: K \longrightarrow$ $K$ where $i \in I=\{1,2, \ldots, m\}$. be finite family of continous nonlinear maps in $K$ such that $F=\bigcap_{i=1}^{m} F\left(T_{i}\right) \neq$ $\emptyset$ and let $\left\{x_{n}\right\}_{n \geq 1}$ be a sequence of successive approximation satisfying

(1) $\lim _{n \rightarrow \infty}\left\|x_{n}-T_{i} x_{n}\right\|=0 \quad \forall i \in I$ 
(2) $\left\|x_{n+1}-x^{*}\right\| \leq\left(1+\tau_{n}\right)\left\|x_{n}-x^{*}\right\|+\nu_{n}$

where $\sum_{n \geq 0} \nu_{n}<\infty$ and $\sum_{n \geq 0} \tau_{n}<\infty$. Then, $\left\{x_{n}\right\}$ converges strongly to a common fixed point of $T_{i}$ 's if and only if $\lim _{n \rightarrow \infty} d\left(x_{n}, F\right)=0$.

Proof Now,

$$
d\left(x_{n+1}, F\right) \leq\left(1+\tau_{n}\right) d\left(x_{n}, F\right)+\nu_{n}
$$

hence $\liminf _{n \rightarrow \infty} d\left(x_{n}, F\right)=0 \Rightarrow \lim _{n \rightarrow \infty} d\left(x_{n}, F\right)$. Also, $\forall k>0$

$$
\begin{aligned}
\left\|x_{n+k+1}-x^{*}\right\| & \leq \Pi_{j=0}^{k}\left(1+\tau_{n+j}\right)\left\|x_{n}-x^{*}\right\|+\sum_{j=0}^{k} \nu_{n+k-j} \Pi_{r=0}^{j-1}\left(1+\tau_{n+k-r}\right) \\
& \leq \Pi_{j=0}^{k}\left(1+\tau_{n+j}\right)\left(\left\|x_{n}-x^{*}\right\|+\sum_{j=0}^{k} \nu_{n+k-j}\right) \\
& \leq Q\left(\left\|x_{n}-x^{*}\right\|+\sum_{j=0}^{k} \nu_{n+j}\right)
\end{aligned}
$$

So that given any $\varepsilon>0$ there exists an integer $n_{0}>0$, such that for all $n \geq n_{0}, d\left(x_{n}, F\right)<\frac{\varepsilon}{4(Q+1)}$ and $\nu_{n+j}<\frac{\varepsilon}{4 m(Q+1)} \forall j=1,2, \ldots, m$. So $\exists x^{*} \in F$ such that $d\left(x_{n_{0}}, x^{*}\right)<\frac{\varepsilon}{4(Q+1)}$ that is, $\left\|x_{n_{0}}-x^{*}\right\|<\frac{\varepsilon}{4(Q+1)}$ Now,

$$
\begin{aligned}
\left\|x_{n+k}-x_{n}\right\| \leq\left\|x_{n+k}-x^{*}\right\|+\left\|x_{n}-x^{*}\right\| & \leq 2 Q\left(\left\|x_{n_{0}}-x^{*}\right\|+\sum_{j=0}^{k} \nu_{n_{0}+j}\right) \\
& \leq 2 Q\left(\frac{\varepsilon}{4(Q+1)}+m \frac{\varepsilon}{4 m(Q+1)}\right) \\
& =2 Q\left(\frac{\varepsilon}{2(Q+1)}<\varepsilon .\right.
\end{aligned}
$$

So, $\left\{x_{n}\right\}$ is a Cauchy sequence in $E$ and so it converges to some $u^{*} \in K$. But, $x_{n}-T_{i} x_{n} \rightarrow 0$ as $n \rightarrow \infty \forall i$ and $T_{i}$ is continuous $\forall i$. Hence, $0=\lim _{n \rightarrow \infty}\left(x_{n}-T_{i} x_{n}\right)=\lim _{n \rightarrow \infty} x_{n}-T_{i}\left(\lim _{n \rightarrow \infty} x_{n}\right)=u^{*}-T_{i} u *$. So that $u^{*} \in F$. i.e $u^{*}=x^{*} \in F$. Hence, $x_{n} \rightarrow x^{*}$ as $n \rightarrow \infty$. This completes the proof since the other part is obvious.

Lemma 2.7. Let $K$ be a closed convex nonempty subset of a Banach space $E$ and let $T_{i}: K \longrightarrow K$ where $i \in$ $I=\{1,2, \ldots, m\}$. be finite family of continuous nonlinear maps in $K$ such that $F=\bigcap_{i=1}^{m} F\left(T_{i}\right) \neq \emptyset$ and let $\left\{x_{n}\right\}_{n \geq 1}$ be a sequence of successive approximation satisfying

(1) $\lim _{n \rightarrow \infty}\left\|x_{n}-T_{i} x_{n}\right\|=0 \quad \forall i \in I$

(2) $\left\|x_{n+1}-x^{*}\right\| \leq\left(1+\tau_{n}\right)\left\|x_{n}-x^{*}\right\|+\nu_{n}$

where $\sum_{n \geq 0} \nu_{n}<\infty$ and $\sum_{n \geq 0} \tau_{n}<\infty$ Then, $\left\{x_{n}\right\}$ converges strongly to a common fixed point of $T_{i}$ 's if one of the $T_{i}$ 's satisfy condition $B$. 
Proof Let $T_{i_{0}}$ satisfy condition B. Then $\exists f:[0, \infty) \rightarrow[0, \infty)$ with $f(0)=0$ and

$$
f\left(d\left(x_{n}, F\right)\right) \leq\left\|x_{n}-T_{i_{0}} x_{n}\right\|
$$

hence

$$
\lim _{n \rightarrow \infty} f\left(d\left(x_{n}, F\right)\right) \leq \lim _{n \rightarrow \infty}\left\|x_{n}-T_{i_{0}} x_{n}\right\|=0 .
$$

Therefore,

$$
\lim _{n \rightarrow \infty} d\left(x_{n}, F\right)=0
$$

Thus by Lemma 2.6, $\left\{x_{n}\right\}$ converges strongly to a common fixed point of $T_{i}$ 's, this concludes the proof.

Lemma 2.8. Let $K$ be a closed convex nonempty subset of a Banach space $E$ and let $T_{i}: K \longrightarrow K$ where $i \in$ $I=\{1,2, \ldots, m\}$. be finite family of continuous nonlinear maps in $K$ such that $F=\bigcap_{i=1}^{N} F\left(T_{i}\right) \neq \emptyset$. Suppose the sequence $\left\{x_{n}\right\}_{n \geq 1}$ of successive approximation satisfies the following conditions

(1) $\lim _{n \rightarrow \infty}\left\|x_{n}-x^{*}\right\|$ exists with $x^{*} \in F$,

(2) $\lim _{n \rightarrow \infty}\left\|x_{n}-T_{i} x_{n}\right\|=0 \quad \forall i \in I$,

(3) $\left\{x_{n}\right\}_{n \geq 1}$ has a convergent subsequence $\left\{x_{n_{j}}\right\}_{n \geq 1}$.

Then, $\left\{x_{n}\right\}$ converges strongly to a point of $F$.

Proof Suppose that $\left\{x_{n}\right\}$ has a convergent subsequence $\left\{x_{n_{j}}\right\}$ and let $x_{n_{j}} \rightarrow p$ as $j \rightarrow \infty$, since $x_{n}-T_{i} x_{n} \rightarrow 0$ as $n \rightarrow \infty$ for all $i \in\{1,2, \ldots, N\}$. It implies that $x_{n_{j}}-T_{i} x_{n_{j}} \rightarrow 0$ as $j \rightarrow \infty$ for all $i \in I$. Also, by continuity of $T_{i} \cdot T_{i} x_{n_{j}} \rightarrow T_{i} p$ as $j \rightarrow \infty$ for all $i \in I$. So, $\left\|p-T_{i} p\right\|=\lim _{n \rightarrow \infty}\left\|x_{n_{j}}-T_{i} x_{n_{j}}\right\|=0, \forall i$ which implies that $p \in F$. Now, $\lim _{n \rightarrow \infty}\left\|x_{n}-p\right\|$ exists from our hypothesis and $\lim _{n \rightarrow \infty}\left\|x_{n_{j}}-p\right\|=0$, so $\lim _{n \rightarrow \infty}\left\|x_{n}-p\right\|=0$. Thus, $\left\{x_{n}\right\}$ converges strongly to a point of $F$.

Remark 2.1. The conditions for which $\left\{x_{n}\right\}$ has a convergent subsequence includes

(1) $T_{i}$ is completely continuous $\forall i \in\{1, \ldots, N\}$.

(2) $T_{i}$ is demicompact $\forall i \in\{1, \ldots, N\}$.

(3) $T_{i}$ is semicompact for some $i \in\{1, \ldots, N\}$.

(4) $K$ is compact.

(5) $K$ is boundedly compact.

Proposition 2.1. Let $K$ be a nonexpansive retract of a uniformly convex Banach space $X$ with nonexpansive retraction $P$. Let $T: K \longrightarrow X$ uniformly continuous generalised asymptotically nonexpansive map with associated sequences $\left\{\mu_{n}\right\}_{n \geq 1},\left\{\eta_{n}\right\}_{n \geq 1} \subset[0,+\infty)$ with $\sum_{n=0}^{\infty}\left(\mu_{n}-1\right)<\infty \sum_{n=0}^{\infty} \eta_{n}<\infty$, suppose that $F(T) \neq \emptyset$. Then $F(T)$ is closed and convex (where $F(T)$ is the fixed point set of $T$ ). 
Proof. Let $\left\{x_{n}\right\}$ be a sequence in $F(T)$ converging to $x^{*} \in K$, then $x_{n}=T x_{n} \forall n \geq 0$. By continuity of $T, x^{*}=\lim _{n \rightarrow \infty} x_{n}=\lim _{n \rightarrow \infty} T x_{n}=T\left(\lim _{n \rightarrow \infty} x_{n}\right)=T x^{*}$. Thus, $x^{*} \in F(T)$ and $F(T)$ is closed. Next, we show that $F(T)$ is convex. For $t \in(0,1)$ and $x, y \in F(T)$, put $z=(1-t) x+t y$, we show that $z=T z$.

$$
\begin{aligned}
& \left\|z-T\left(P_{K} T\right)^{n-1} z\right\|^{2}=\|z\|^{2}-2\left\langle z, T\left(P_{K} T\right)^{n-1} z\right\rangle+\left\|T\left(P_{K} T\right)^{n-1} z\right\|^{2} \\
& =\|z\|^{2}-2(1-t)\left\langle x, T\left(P_{K} T\right)^{n-1} z\right\rangle-2 t\left\langle y, T\left(P_{K} T\right)^{n-1} z\right\rangle \\
& +\left\|T\left(P_{K} T\right)^{n-1} z\right\|^{2} \\
& =\|z\|^{2}+(1-t)\left\|x-T\left(P_{K} T\right)^{n-1} z\right\|^{2}+t\left\|y-T\left(P_{K} T\right)^{n-1} z\right\|^{2} \\
& -(1-t)\|x\|^{2}-t\|y\|^{2} \\
& \leq\|z\|^{2}+(1-t)\left(\mu_{n}\|x-z\|+\eta_{n}\right)^{2}+t\left(\mu_{n}\|y-z\|+\eta_{n}\right)^{2} \\
& -(1-t)\|x\|^{2}-t\|y\|^{2} \\
& =\|z\|^{2}+(1-t)\left(\mu_{n}^{2}\|x-z\|^{2}+\left(2 \mu_{n}\|x-z\|+\eta_{n}\right) \eta_{n}\right) \\
& +t\left(\mu_{n}^{2}\|y-z\|^{2}+\left(2 \mu_{n}\|y-z\|+\eta_{n}\right) \eta_{n}\right)-(1-t)\|x\|^{2}-t\|y\|^{2} \\
& =\|z\|^{2}+(1-t) \mu_{n}^{2}\left(\|x\|^{2}-\|z\|^{2}-2\langle x, z\rangle\right) \\
& +t \mu_{n}^{2}\left(\|y\|^{2}-\|z\|^{2}-2\langle y, z\rangle\right) \\
& +2 \mu_{n} \eta_{n}((1-t)\|x-z\|+t\|y-z\|)+\eta_{n}^{2}-(1-t)\|x\|^{2}-t\|y\|^{2} \\
& =\left(1+\mu_{n}^{2}\right)\|z\|^{2}+\left(\mu_{n}^{2}-1\right)\left((1-t)\|x\|^{2}+t\|y\|^{2}\right)-2 \mu_{n}((1-t)\langle x, z\rangle \\
& +t\langle y, z\rangle)+2 \mu_{n} \eta_{n}((1-t)\|x-z\|+t\|y-z\|)+\eta_{n}^{2} \\
& \leq\left(\mu_{n}^{2}-1\right)\left((1-t)\|x\|^{2}+t\|y\|^{2}+\|z\|^{2}\right)+2 \mu_{n}^{2}\|z\|^{2} \\
& -2 \mu_{n}^{2}((1-t)\langle x, z\rangle+t\langle y, z\rangle) \\
& +2 \mu_{n} \eta_{n}((1-t)\|x-z\|+t\|y-z\|)+\eta_{n}^{2} \\
& =\left(\mu_{n}^{2}-1\right)\left((1-t)\|x\|^{2}+t\|y\|^{2}+\|z\|^{2}\right)-2 \mu_{n}^{2}((1-t)\langle x, z\rangle \\
& \left.+t\langle y, z\rangle-(1-t)\|z\|^{2}-t\|z\|^{2}\right) \\
& +2 \mu_{n} \eta_{n}((1-t)\|x-z\|+t\|y-z\|)+\eta_{n}^{2} \\
& =\left(\mu_{n}^{2}-1\right)\left((1-t)\|x\|^{2}+t\|y\|^{2}+\|z\|^{2}\right)-2 \mu_{n}^{2}((1-t)\langle x-z, z\rangle \\
& +t\langle y-z, z\rangle)+2 \mu_{n} \eta_{n}((1-t)\|x-z\|+t\|y-z\|)+\eta_{n}^{2} \\
& =\left(\mu_{n}^{2}-1\right)\left((1-t)\|x\|^{2}+t\|y\|^{2}+\|z\|^{2}\right)-2 \mu_{n}^{2}\langle(1-t)(x-z)+t(y-z), z\rangle \\
& +2 \mu_{n} \eta_{n}((1-t)\|x-z\|+t\|y-z\|)+\eta_{n}^{2}
\end{aligned}
$$




$$
\begin{aligned}
= & \left(\mu_{n}^{2}-1\right)\left((1-t)\|x\|^{2}+t\|y\|^{2}+\|z\|^{2}\right)-2 \mu_{n}^{2} t(1-t)(\langle x-y+y-x, z\rangle \\
& +\eta_{n}^{2}+2 \mu_{n} \eta_{n}((1-t)\|x-z\|+t\|y-z\|) \\
= & \left(\mu_{n}^{2}-1\right)\left((1-t)\|x\|^{2}+t\|y\|^{2}+\|z\|^{2}\right)+4 t(1-t)\|x-y\| \mu_{n} \eta_{n}+\eta_{n}^{2} .
\end{aligned}
$$

Thus, $\lim _{n \rightarrow \infty}\left\|z-T\left(P_{K} T\right)^{n-1} z\right\|=0$, which implies that $\lim _{n \rightarrow \infty} T\left(P_{K} T\right)^{n-1} z=z$ and hence $z=$ $\lim _{n \rightarrow \infty} T\left(P_{K} T\right)^{n-1} z=T P_{K}\left(\lim _{n \rightarrow \infty} T\left(P_{K} T\right)^{n-2} z\right)=T P_{K} z=T z$. Thus, $z \in F(T)$. This completes the proof.

Proposition 2.2. Let $K$ be a nonexpansive retract of a uniformly convex Banach space $X$ with nonexpansive retraction $P$. Let $T_{i}: K \longrightarrow X(i=1, \ldots, m)$ be a finite family of uniformly continuous generalised asymptotically nonexpansive map with associated sequences $\left\{\mu_{i n}\right\}_{n \geq 1},\left\{\eta_{i n}\right\}_{n \geq 1} \subset[0,+\infty)$ with $\sum_{n=0}^{\infty}\left(\mu_{i n}-1\right)<\infty, \sum_{n=0}^{\infty} \eta_{i n}<\infty$. Suppose that $F=\bigcap_{i=1}^{m} F\left(T_{i}\right) \neq \emptyset$. Then $F$ is closed and convex.

Proof. By Lemma 2.1, we have that $F\left(T_{i}\right)$ is closed for each $i$. Now, $F=\bigcap_{i=1}^{m} F\left(T_{i}\right)$ is a finite intersection of closed sets, hence closed. Also, by Lemma 2.1, we have that $F\left(T_{i}\right)$ is convex for each $i$. Since $F=\bigcap_{i=1}^{m} F\left(T_{i}\right)$ is a finite intersection of convex set, we have that $F$ is convex.

Proposition 2.3. Suppose that there exist $c>0, k>0$ constants such that $\phi(t) \leq c t$ for all $\geq k$, then $T$ is total asymptotically nonexpansive if and only if $T$ is generalised asymptotically nonexpansive.

Proof It is known that every generalised asymptotically nonexpansive map is total asymptotically nonexpansive, so it suffices to show that that every total asymptotically nonexpansive with the condition of our hypothesis is generalised asymptotically nonexpansive. Now, let $T$ be such that

$$
\left\|T^{n} x-T^{n} y\right\| \leq\|x-y\|+\mu_{n} \phi(\|x-y\|)+\eta_{n} \quad n \geq 1
$$

Since $\phi$ is continuous, it follows that $\phi$ reaches its maximum (say $c_{0}$ ) on the interval $[0, k]$; moreover, $\phi(t) \leq c t$ whenever $t>k$. Thus,

$$
\phi(t) \leq c_{0}+c t \forall t \in[0,+\infty) .
$$

So, we have,

$$
\begin{aligned}
\left\|T^{n} x-T^{n} y\right\| & \leq\|x-y\|+\mu_{n}\left(c_{0}+c\|x-y\|\right)+\eta_{n} \quad n \geq 1 \\
& =\left(1+\mu_{n} c\right)\|x-y\|+\mu_{n} c_{0}+\eta_{n} \\
& =\left(1+\nu_{n}\right)\|x-y\|+\gamma_{n}
\end{aligned}
$$

where $\nu_{n}=\mu_{n} c$ and $\gamma_{n}=\mu_{n} c_{0}+\eta_{n}$. This completes the proof. 
Corollary 2.1. Let $K$ be a nonexpansive retract of a uniformly convex Banach space $X$ with nonexpansive retraction $P$. Let $T: K \longrightarrow X$ be a uniformly continuous total asymptotically nonexpansive map with associated sequences $\left\{\mu_{n}\right\}_{n \geq 1},\left\{\eta_{n}\right\}_{n \geq 1} \subset[0,+\infty)$ with $\sum_{n=0}^{\infty}\left(\mu_{n}-1\right)<\infty \sum_{n=0}^{\infty} \eta_{n}<\infty$. Suppose that there exist $c>0, k>0$ constants such that $\phi(t) \leq c t \forall t \geq k$, and that $F(T) \neq \emptyset$ then $F(T)$ is closed and convex.

Corollary 2.2. Let $K$ be a nonexpansive retract of a uniformly convex Banach space $X$ with nonexpansive retraction $P$. Let $T_{i}: K \longrightarrow X(i=1, \ldots, m)$ be a finite family of uniformly continuous total asymptotically nonexpansive maps with associated sequences $\left\{\mu_{i n}\right\}_{n \geq 1},\left\{\eta_{i n}\right\}_{n \geq 1} \subset[0,+\infty)$ with $\sum_{n=0}^{\infty}\left(\mu_{i n}-1\right)<\infty \sum_{n=0}^{\infty} \eta_{i n}<$ $\infty$. Suppose that there exist $c>0, k>0$ constants such that $\phi(t) \leq c t$ for all $t \geq k$, and that $F=$ $\bigcap_{i=1}^{m} F\left(T_{i}\right) \neq \emptyset$ then $F$ is closed and convex.

\section{Main RESUlts}

Proposition 3.1. Let $H$ be a normed linear space, let $K$ be a closed convex nonempty subset of $H$ and let $T_{i} K \longrightarrow H(i \in I=\{1, \ldots, m\})$ be a finite family of continuous generalised asymptotically nonexpansive map with sequences $\left\{\mu_{i n}\right\}_{n \geq 1},\left\{\eta_{i n}\right\}_{n \geq 1} \subset[0,+\infty)$ such that $\lim _{n \rightarrow \infty} \mu_{\text {in }}=1$ and $\lim _{n \rightarrow \infty} \eta_{n}=0$ with

$$
\sum_{n=0}^{\infty}\left(\mu_{i n}-1\right)<\infty \sum_{n=0}^{\infty} \eta_{i n}<\infty
$$

. Suppose that $F(T) \neq \emptyset$ and let $\left\{x_{n}\right\}_{n \geq 1}$ be a sequence generated iteratively by starting with an arbitrary $x_{0} \in K$, define by

$$
\begin{aligned}
& y_{n, i}=P_{K}\left[\left(1-\alpha_{n}\right) x_{n}+\alpha_{n} T_{i}\left(P T_{i}\right)^{n-1} y_{n, i-1}\right], \\
& y_{n, 0}=x_{n} ; y_{n, m}=x_{n+1}=y_{n+1} \quad n \geq 0
\end{aligned}
$$

where $\left\{\alpha_{n}\right\}_{n \geq 1}$, is a sequence in $(0,1)$ such that $0<\zeta<\beta_{n}<\epsilon<1 \forall n \geq 1$. Let $x^{*} \in F$, then $\lim _{n \rightarrow \infty}\left\|x_{n}-x^{*}\right\|$ exist

Proof. Let $x^{*} \in F, j \in I$ then from (3.1) we have that

$$
\begin{aligned}
\left\|y_{n, j}-x^{*}\right\| & =\left\|P_{K}\left[\left(1-\alpha_{n}\right) x_{n}+\alpha_{n} T_{j}\left(P T_{j}\right)^{n-1} y_{n, j-1}\right]-P x^{*}\right\| \\
& \leq\left(1-\alpha_{n}\right)\left\|x_{n}-x^{*}\right\|+\alpha_{n}\left\|T_{j}\left(P T_{j}\right)^{n-1} y_{n, j-1}-x^{*}\right\| \\
& \left.\leq\left(1-\alpha_{n}\right)\left\|x_{n}-x^{*}\right\|+\alpha_{n}\left(\mu_{n, j}\right)\left\|y_{n, j-1}-x^{*}\right\|+\eta_{i n}\right) . \\
& =\left(1-\alpha_{n}\right)\left\|x_{n}-x^{*}\right\|+\alpha_{n} \mu_{n, j}\left(\left\|y_{n, j-1}-x^{*}\right\|+\alpha_{n} \eta_{n, j}\right)
\end{aligned}
$$




$$
\begin{gathered}
\left\|y_{n, 1}-x^{*}\right\| \leq\left(1-\alpha_{n}\right)\left\|x_{n}-x^{*}\right\|+\alpha_{n} \mu_{n, 1}\left(\left\|y_{n, 0}-x^{*}\right\|+\alpha_{n} \eta_{n, 1}\right) \\
\leq\left(1+\alpha_{n}\left(\mu_{n, 1-1}\right)\right)\left\|x_{n}-x^{*}\right\|+\alpha_{n} \eta_{n, 1} \\
\left\|y_{n, 2}-x^{*}\right\| \leq \quad\left(1-\alpha_{n}\right)\left\|x_{n}-x^{*}\right\|+\alpha_{n} \mu_{n, 2}\left((1+\alpha(\mu n, 1-1))\left\|x_{n}-x^{*}\right\|+\alpha_{n} \eta_{n, 1}\right)+\alpha_{n} \eta_{n, 2} . \\
\left.\leq \quad\left(1+\alpha_{n}\left(\mu_{n, 2}-1\right)+\alpha_{n}^{2} \mu_{(} n, 2\right)\left(\mu_{n, 1}-1\right)\right)\left\|x_{n}-x^{*}\right\|+\alpha_{n}^{2} \mu_{n, 2} \eta_{n, 1}+\alpha_{n} \eta_{n, 2} . \\
\left\|y_{n, 3}-x^{*}\right\| \leq \\
\left.\quad\left(1+\alpha_{n}\left(\mu_{n, 3}-1\right)+\alpha_{n}^{2} \mu(n, 3)\left(\mu_{n, 2}-1\right)+\alpha_{n}^{3} \mu n, 3\right) \mu_{n, 2}\left(\mu_{n, 1}-1\right)\right)\left\|x_{n}-x^{*}\right\| \\
\quad+\alpha_{n} \eta_{n, 3}+\alpha_{n}^{2} \mu_{n, 3} \eta_{n, 2}+\alpha_{n}^{3} \mu_{n, 3} \mu_{n, 2} \eta_{n, 1} .
\end{gathered}
$$

Hence,

$$
\begin{aligned}
\left\|y_{n, j}-x^{*}\right\| \leq & \left(1+\sum_{t=1}^{j} \alpha_{n}^{t} \Pi_{s=1}^{t-1} \mu_{n, j-s+1}\left(\mu_{n, j-t+1}-1\right)\right)\left\|x_{n}-x^{*}\right\| \\
& +\sum_{t=1}^{j} \alpha_{n}^{t} \Pi_{s=1}^{t-1} \mu_{n, j-s+1} \eta_{n, j-t+1} . \\
\left\|x_{n+1}-x^{*}\right\| \leq & \left(1+\sum_{t=1}^{m} \alpha_{n}^{t} \Pi_{s=1}^{t-1} \mu_{n, m-s+1}\left(\mu_{n, m-t+1}-1\right)\right)\left\|x_{n}-x^{*}\right\| \\
& +\sum_{t=1}^{m} \alpha_{n}^{t} \Pi_{s=1}^{t-1} \mu_{n, m-s+1} \eta_{n, m-t+1} \\
\leq & \left(1+q^{m-1} b \sum_{j=1}^{m}\left(\mu_{n, j}-1\right)\right)\left\|x_{n}-x^{*}\right\|+q^{m-1} b \sum_{j=1}^{m} \eta_{n, j} .
\end{aligned}
$$

(since there exists $n_{0}$ such that $\mu_{n, i} \leq q$ for all $n \geq n_{0}, \forall j \in I$ ) So, $\lim _{n \rightarrow \infty}\left\|x_{n}-x^{*}\right\|$ exist; and hence $\left\{x_{n}\right\},\left\{y_{n, j}\right\}$ are bounded.

Theorem 3.1. Let $K$ be a nonexpansive retract of a uniformly convex Banach space $X$ with nonexpansive retraction $P$. Let $T_{i}: K \longrightarrow E$ be a finite family of uniformly continuous generalised asymptotically nonexpansive maps with sequences $\left\{\mu_{i n}\right\}_{n \geq 1},\left\{\eta_{i n}\right\}_{n \geq 1} \subset[0,+\infty)$ such that

$$
\lim _{n \rightarrow \infty} \mu_{i n}=1, \lim _{n \rightarrow \infty} \eta_{i n}=0, \sum_{n=0}^{\infty}\left(\mu_{i n}-1\right)<\infty \sum_{n=0}^{\infty} \eta_{i n}<\infty
$$


Suppose that $F=\bigcap_{i=1}^{N} F\left(T_{i}\right)$ is not empty and let $\left\{x_{n}\right\}_{n \geq 1}$ be a sequence generated iteratively by (3.1) where $\left\{\alpha_{n}\right\}_{n \geq 1}$ is a sequence in $(0,1)$ satisfying the following conditions:

$$
\sum_{n=1}^{\infty} \alpha_{n}<\infty, 0<\zeta<\alpha_{n}<\epsilon<1 \forall n \geq 1
$$

, then $\forall j \in\{1,2, \ldots, m\}, \lim _{n \rightarrow \infty}\left\|x_{n}-T_{j} x_{n}\right\|=0$ and $\left\{x_{n}\right\}_{n \geq 1}$ converges weakly to a point of $F$.

Proof. Let $x^{*} \in F$

$$
\begin{aligned}
\left\|y_{n, j}-x^{*}\right\|^{2} \leq & \left(1-\alpha_{n}\right)\left\|x_{n}-x^{*}\right\|^{2}+\alpha_{n}\left\|T_{j}\left(P T_{j}\right)^{n-1} y_{n, j-1}-x^{*}\right\| \\
& -\alpha_{n}\left(1-\alpha_{n}\right) g\left(\left\|x_{n}-T_{j}\left(P T_{j}\right)^{n-1} y_{n, j-1}\right\|\right) \\
\leq & \left(1-\alpha_{n}\right)\left\|x_{n}-x^{*}\right\|^{2}+\alpha_{n}\left(\mu_{n, j}\left\|y_{n, j-1}-x^{*}\right\|+\eta_{n, j}\right)^{2} \\
& -\alpha_{n}\left(1-\alpha_{n}\right) g\left(\left\|x_{n}-T_{j}\left(P T_{j}\right)^{n-1} y_{n, j-1}\right\|\right) \\
\leq & \left(1-\alpha_{n}\right)\left\|x_{n}-x^{*}\right\|^{2}+\alpha_{n} \mu_{n, j}^{2}\left\|y_{n, j-1}-x^{*}\right\|^{2} \\
& +\alpha_{n}\left(2 \mu_{n, j}\left\|y_{n, j-1}-x^{*}\right\|+\eta_{n, j}\right) \eta_{n, j}-\alpha_{n}\left(1-\alpha_{n}\right) g\left(\left\|x_{n}-T_{j}\left(P T_{j}\right)^{n-1} y_{n, j-1}\right\|\right)
\end{aligned}
$$

So

$$
\begin{aligned}
\left\|y_{n, 1}-x^{*}\right\|^{2} \leq & \left(1+\alpha_{n}\left(\mu_{n, 1}^{2}-1\right)\right)\left\|x_{n}-x^{*}\right\|^{2}+\alpha_{n}\left(2 \mu_{n, 1}\left\|x_{n}-x^{*}\right\|+\eta_{n, 1}\right) \eta_{n, 1} \\
& -\alpha_{n}\left(1-\alpha_{n}\right) g\left(\left\|x_{n}-T_{1}\left(P T_{1}\right)^{n-1} x_{n}\right\|\right) \\
\left\|y_{n, 2}-x^{*}\right\|^{2} \leq & \left(1+\alpha_{n}\left(\mu_{n, 2}^{2}-1\right)+\alpha_{n}^{2} \mu_{n, 2}^{2}\left(\mu_{n, 1}^{2}-1\right)\right)\left\|x_{n}-x^{*}\right\|^{2} \\
& +\alpha_{n}\left(2 \mu_{n, 2}\left\|y_{n, 1}-x^{*}\right\|+\eta_{n, 2}\right) \eta_{n, 2}+\alpha_{n}^{2} \mu_{n, 2}^{2}\left(2 \mu_{n, 1}\left\|x_{n}-x^{*}\right\|+\eta_{n, 1}\right) \eta_{n, 1} \\
& -\alpha_{n}\left(1-\alpha_{n}\right) g\left(\left\|x_{n}-T_{2}\left(P T_{2}\right)^{n-1} y_{n, 1}\right\|\right) \\
& -\alpha_{n}^{2} \mu_{n, 2}^{2}\left(1-\alpha_{n}\right) g\left(\left\|x_{n}-T_{1}\left(P T_{1}\right)^{n-1} x_{n}\right\|\right)
\end{aligned}
$$

So,

$$
\begin{aligned}
\left\|y_{n, j}-x^{*}\right\|^{2} \leq & \left(1+\sum_{t=1}^{j} \alpha_{n}^{t} \Pi_{s=1}^{t-1} \mu_{n, j-s+1}^{2}\left(\mu_{n, j-t+1}^{2}-1\right)\right)\left\|x_{n}-x^{*}\right\|^{2} \\
& +\sum_{t=1}^{j} \alpha_{n}^{t} \Pi_{s=1}^{t-1} \mu_{n, j-s+1}^{2}\left(2 \mu_{n, j-t+1}\left\|y_{n, j-t-1}-x^{*}\right\|+\eta_{n, j-t+1}\right) \eta_{n, j-t+1} \Pi_{s=0}^{t-1} \mu_{n, j-s}^{2} \\
& -\left(1-\alpha_{n}\right) \sum_{t=1}^{j} \alpha_{n}^{t} g\left(\left\|x_{n}-T_{j-t+1}\left(P T_{j-t+1}\right)^{n-1} y_{n, j-t}\right\|\right) \Pi_{s=1}^{t-1} \mu_{n, j-s+1}^{2}
\end{aligned}
$$


Hence,

$$
\begin{aligned}
\left\|x_{n+1}-x^{*}\right\|^{2} \leq & \left(1+\sum_{t=1}^{m} \alpha_{n}^{t} \Pi_{s=1}^{t-1} \mu_{n, m-s+1}^{2}\left(\mu_{n, m-t+1}^{2}-1\right)\right)\left\|x_{n}-x^{*}\right\|^{2} \\
& +\sum_{t=1}^{m} \alpha_{n}^{t} \Pi_{s=1}^{t-1} \mu_{n, m-s+1}^{2}\left(2 \mu_{n, m-t+1}\left\|y_{n, m-t}-x^{*}\right\|+\eta_{n, m-t+1}\right) \eta_{n, m-t+1} \\
& -\left(1-\alpha_{n}\right) \sum_{t=1}^{m} \alpha_{n}^{t} \Pi_{s=1}^{t-1} \mu_{n, m-s+1}^{2} g\left(\left\|x_{n}-T_{m-t+1}\left(P T_{m-t+1}\right)^{n-1} y_{n, m-t}\right\|\right) \\
\leq & \left(1+q^{2(m-1)} b \sum_{j=1}^{m}\left(\mu_{n, j}^{2}-1\right)\right)\left\|x_{n}-x^{*}\right\|+q^{2(m-1)} b \sum_{j=1}^{m}\left(2 \mu_{n, j}\left\|y_{n, j-1}-x^{*}\right\|\right. \\
& \left.+\eta_{n, j}\right) \eta_{n, j} \|-a^{m}\left(1-\alpha_{n}\right) \sum_{j=1}^{m} g\left(\left\|x_{n}-T_{m-j+1}\left(P T_{m-j+1}\right)^{n-1} y_{n, m-j}\right\|\right) \\
\leq & \left(1+q^{2(m-1)} b \sum_{j=1}^{m}\left(\mu_{n, j}^{2}-1\right)\right)\left\|x_{n}-x^{*}\right\| \\
& +q^{2(m-1)} b \sum_{j=1}^{m} \eta_{n, j}-a^{m}\left(1-\alpha_{n}\right) \sum_{j=1}^{m} g\left(\left\|x_{n}-T_{m-j+1}\left(P T_{m-j+1}\right)^{n-1} y_{n, m-j}\right\|\right)
\end{aligned}
$$

So,

$$
\begin{aligned}
\left\|x_{n+1}-x^{*}\right\|^{2} \leq & \left(1+d_{0} \sum_{j=1}^{m}\left(\mu_{n, j}^{2}-1\right)\right)\left\|x_{n}-x^{*}\right\| \\
& +d_{1} \sum_{j=1}^{m} \eta_{n, j}-d_{2} \sum_{j=1}^{m} g\left(\left\|x_{n}-T_{j}\left(P T_{j}\right)^{n-1} y_{n, j-1}\right\|\right)
\end{aligned}
$$

So, $\lim _{n \rightarrow \infty} g\left(\left\|x_{n}-T_{j}\left(P T_{j}\right)^{n-1} y_{n, j-1}\right\|\right)=0$, thus $\lim _{n \rightarrow \infty}\left\|x_{n}-T_{j}\left(P T_{j}\right)^{n-1} y_{n, j-1}\right\|=0 \forall j=1, \ldots, m$. Now,

$$
\begin{aligned}
\left\|x_{n}-T_{j}\left(P T_{j}\right)^{n-1} x_{n}\right\| & \leq\left\|x_{n}-T_{j}\left(P T_{j}\right)^{n-1} y_{n, j-1}\right\|+\left\|T_{j}\left(P T_{j}\right)^{n-1} y_{n, j-1}-T_{j}\left(P T_{j}\right)^{n-1} x_{n}\right\| \\
& \leq\left\|x_{n}-T_{j}\left(P T_{j}\right)^{n-1} y_{n, j-1}\right\|+\mu_{n, j}\left\|y_{n, j-1}-x_{n}\right\|+\eta_{n, j} \\
& \leq\left\|x_{n}-T_{j}\left(P T_{j}\right)^{n-1} y_{n, j-1}\right\|+\mu_{n, j} \alpha_{n}\left\|x_{n}-T_{j-1}\left(P T_{j-1}\right)^{n-1} y_{n, j-2}\right\|+\eta_{n, j}
\end{aligned}
$$

Hence, $\lim _{n \rightarrow \infty}\left\|x_{n}-T_{j}\left(P T_{j}\right)^{n-1} x_{n}\right\|=0 \forall j=1, \ldots, m$.

Further, $\left\|x_{n}-T_{j} x_{n}\right\| \leq\left\|x_{n}-T_{j}\left(P T_{j}\right)^{n-1} y_{n, j-1}\right\|+\left\|T_{j}\left(P T_{j}\right)^{n-1} y_{n, j-1}-T_{j} x_{n}\right\|$

$$
\begin{aligned}
\left\|\left(P T_{j}\right)^{n-1} y_{n, j-1}-x_{n}\right\| \leq & \left\|T_{j}\left(P T_{j}\right)^{n-2} y_{n, j-1}-x_{n}\right\| \\
\leq & \left\|T_{j}\left(P T_{j}\right)^{n-2} y_{n, j-1}-T_{j}\left(P T_{j}\right)^{n-2} y_{n-1, j-1}\right\| \\
& +\left\|T_{j}\left(P T_{j}\right)^{n-2} y_{n-1, j-1}-x_{n-1}\right\|+\left\|x_{n-1}-x_{n}\right\| \\
\leq & \mu_{n-1, j}\left\|y_{n, j-1}-y_{n-1, j-1}\right\|+\eta_{n-1, i} \\
& +\left\|x_{n-1}-T_{j}\left(P T_{j}\right)^{n-2} y_{n-1, j-1}\right\|+\left\|x_{n}-x_{n-1}\right\|
\end{aligned}
$$




$$
\begin{aligned}
\left\|y_{n, j}-y_{n-1, j}\right\| \leq & \left\|y_{n, j}-x_{n}\right\|+\left\|x_{n}-x_{n-1}\right\|+\| x_{n-1}-y_{n-1, j} \\
\leq & \alpha_{n}\left\|x_{n}-T_{j}\left(P T_{j}\right)^{n-1} y_{n, j-1}\right\|+\alpha_{n-1}\left\|x_{n-1}-T_{m}\left(P T_{m}\right)^{n-2} y_{n-1, m-1}\right\| \\
& +\alpha_{n-1}\left\|x_{n-1}-T_{j}\left(P T_{j}\right)^{n-2} y_{n-1, j-1}\right\|
\end{aligned}
$$

So, $\lim _{n \rightarrow \infty}\left\|y_{n, j}-y_{n-1, j}\right\|=0$. Also, $\lim _{n \rightarrow \infty}\left\|x_{n}-x_{n-1}\right\|=0$

so that $\lim _{n \rightarrow \infty}\left\|\left(P T_{j}\right)^{n-1} y_{n, j-1}-x_{n}\right\|=0$. Hence $\lim _{n \rightarrow \infty}\left\|x_{n}-T_{j} x_{n}\right\|=0 \forall j=1, \ldots, m$. By reflexivity $\exists z \in K$ and $\left\{x_{n_{j}}\right\} \subset\left\{x_{n}\right\}$ such that, $\left\{x_{n_{j}}\right\} \rightarrow^{w} z$ as $j \rightarrow \infty$. Since, $x_{n_{j}}-T_{i} x_{n_{j}} \rightarrow 0$ as $j \rightarrow \infty \forall i$ then $z \in F\left(T_{i}\right) \forall i$ and so $z \in F=\bigcap_{i=1}^{m} F\left(T_{i}\right)$. Let $\omega_{w}\left(x_{n}\right)$ be subsequential limit set of the sequence $\left\{x_{n}\right\}$. Let $q \in \omega_{w}\left(x_{n}\right)$ arbitrary. Then $\exists\left\{x_{n_{r}}\right\} \subset\left\{x_{n}\right\} \ni\left\{x_{n_{r}}\right\}$ converges weakly q and $x_{n r}-T_{i} x_{n r} \rightarrow 0$ as $r \rightarrow \infty \forall i$. Thus, $\omega_{w}\left(x_{n}\right) \subseteq F$. Thus $\left\{x_{n}\right\}_{n \geq 1}$ converges weakly to a point of $F$.

Theorem 3.2. Let $K, X, P, T_{i}^{\prime} s, F,\left\{x_{n}\right\}$ be as in Theorem 3.1 Then, $\left\{x_{n}\right\}$ converges strongly to a fixed point of $T$ if and only if $\liminf _{n \rightarrow \infty} d\left(x_{n}, F\right)=0$ (where $F=F(T)$ ).

The Proof follows from Lemma 2.6, since from Theorem 3.1 and it's proof, the conditions of the lemma are satisfied.

Theorem 3.3. Let $K, X, P, T_{i}^{\prime} s, F,\left\{x_{n}\right\}$ be as in Theorem 3.1 Then, $\left\{x_{n}\right\}$ converges strongly to a common fixed point of $T_{i}$ 's if one of the $T_{i}$ 's satify condition $B$.

The Proof follows from Lemma 2.7, since from the proof of Theorem 3.1, the conditions of the lemma are satisfied.

Theorem 3.4. Let $K, X, P, T_{i}^{\prime} s, F,\left\{x_{n}\right\}$ be as in Theorem 3.1 Then, $\left\{x_{n}\right\}$ converges strongly to a common fixed point of $T_{i}$ 's if $\left\{x_{n}\right\}_{n \geq 1}$ has a convergent subsequence $\left\{x_{n_{j}}\right\}_{n \geq 1}$

The Proof follows from Lemma 2.8, since from the proof of Theorem 3.1, the conditions of the lemma are satisfied.

As a result of the proposition 2.3 we have the following results.

Theorem 3.5. Let $K$ be a nonexpansive retract of a uniformly convex Banach space $X$ with nonexpansive retraction $P$. Let $T_{i}: K \longrightarrow E$ be a finite family of uniformly continuous total asymptotically nonexpansive maps with sequences $\left\{\mu_{i n}\right\}_{n \geq 1},\left\{\eta_{i n}\right\}_{n \geq 1} \subset[0,+\infty)$ such that

$$
\lim _{n \rightarrow \infty} \mu_{i n}=0=\lim _{n \rightarrow \infty} \eta_{i n}, \sum_{n=0}^{\infty}\left(\mu_{i n}-1\right)<\infty \sum_{n=0}^{\infty} \eta_{i n}<\infty
$$

and with function $\phi:[0,+\infty) \longrightarrow[0,+\infty)$ satisfying $\phi(t) \leq M_{0} t$ for all $t>M_{1}$, for some constants $M_{0}, M_{1}>0$. Suppose that $F=\bigcap_{i=1}^{m} F\left(T_{i}\right)$ is not empty and let $\left\{x_{n}\right\}_{n \geq 1}$ be a sequence generated iteratively 
by (3.1) where $\left\{\alpha_{n}\right\}_{n \geq 1}$ is a sequence in $(0,1)$ satisfying the following conditions:

$$
\sum_{n=1}^{\infty} \alpha_{n}<\infty, 0<\zeta<\alpha_{n}<\epsilon<1 \forall n \geq 1
$$

then for all $j \in\{1,2, \ldots, m\}, \lim _{n \rightarrow \infty}\left\|x_{n}-T_{j} x_{n}\right\|=0$ and $\left\{x_{n}\right\}_{n \geq 1}$ converges weakly to a point of $F$.

Theorem 3.6. Let $K, X, P, T_{i}^{\prime} s, F,\left\{x_{n}\right\}$ be as in Theorem 3.5 Then, $\left\{x_{n}\right\}$ converges strongly to a fixed point of $T$ if and only if $\liminf _{n \rightarrow \infty} d\left(x_{n}, F\right)=0$.

Theorem 3.7. Let $K, X, P, T_{i}^{\prime} s, F,\left\{x_{n}\right\}$ be as in Theorem 3.5. Then, $\left\{x_{n}\right\}$ converges strongly to a common fixed point of $T_{i}$ 's if one of the $T_{i}$ 's satify condition $B$.

Theorem 3.8. Let $K, X, P, T_{i}^{\prime} s, F,\left\{x_{n}\right\}$ be as in Theorem 3.5 Then, $\left\{x_{n}\right\}$ converges strongly to a common fixed point of $T_{i}$ 's if $\left\{x_{n}\right\}_{n \geq 1}$ has a convergent subsequence $\left\{x_{n_{j}}\right\}_{n \geq 1}$.

Our iterative process generalise some of the existing ones, our theorems improves, generalise and extend several known results and our method of proof is of independent interest.

Conflicts of Interest: The author(s) declare that there are no conflicts of interest regarding the publication of this paper.

\section{REFERENCES}

[1] Alber, Y., Chidume, C. E. and Zegeye, H., Approximating Fixed Points of Total Asymptotically Nonexpansive Mappings.Fixed Point Theory Appl., 2006(2006), article ID 10673.

[2] Chidume, C. E.; Geometric properties of Banach spaces and nonlinear iterations. Springer Verlag Series: Lecture Notes in Mathematics Vol. 1965(2009) XVII, 326p.

[3] Goebel, K. and Kirk, W.A., A Fixed Point Theorem for Asymptotically Nonexpansive Mappings. Proc. Amer. Math. Soc. 35(1972), 171-174.

[4] Isiogugu, F.O. and Osilike, M.O., Fixed Point and Convergence Theorems for Certain Classes of Mappings.J. Nigerian Math. Soc., 31(2013), 147-165.

[5] Lim, T.C. and Xu, H.K., Fixed Point Theorems For Asymtotically Nonexpansive Mappings. Nonlinear Anal., Theory Methods Appl., 22(1994), 1345-1355.

[6] Moore, C. and Nnoli, B.V.C., Strong Convergence of Averaged Approximants for Lipschitz Pseudocontrative Maps. J. Math. Anal. Appl., 260(2006), 169-178.

[7] Moore, C. and Ofoedu, E. U., Convergence of Iteration Process to Random Fixed Points of Uniformly L- Lipschitzian Asymptotically Hemi-contractive Random Maps in Banach Spaces. J. Nigerian Math. Soc., 25(2006), 87-94.

[8] Ofoedu, E.U. and Nnubia, A.C., Approximation of minimum-norm fixed point of total asymptotically nonexpansive mapping. Afrika Matematika, 26(2015), 699-715.

[9] Osilike, M.O. and Shehu,Y., Explicit Averaging Cyclic Algorithm for Common Fixed Points of Asymptotically Strictly Pseudocontractive Maps. Appl. Math. Comput., 213(2009), 584-553.

[10] Osilike, M.O. and Shehu,Y., Explicit Averaging Cyclic Algorithm for Common Fixed Points of Asymptotically Strictly Pseudocontractive Maps in Banach Spaces. Computers Math. Appl., 57(2009), 1502-1510. 
[11] Okeke, G.A., Bishop, S. A. and Khan, S.H., Iterative Approximation of Fixed Point of Multivalued $\rho$-Quasi-Nonexpansive Mappings in Modular Function Spaces with Applications. J. Funct. Spaces, 2018(2018), Article ID 1785702.

[12] Takahashi, W., Nonlinear Functional Analysis- Fixed point theory and applications. Yokohanna publisher inc. Yokohanna, (2000).

[13] Zegeye, H. and Shahzad, N., Strong Convergence of an Implicit Iteration Process for a Finite Family of Generalised Asymptotically Quasi- Gonexpansive Maps, Appl. Math. Comput., 189(2007), 1058-1065.

[14] Zegeye, H. and Shahzad N., Approximation of the common minimum-norm fixed point of a finite family of asymptotically nonexpansive mappings, Fixed Point Theory Appl., 2013(2013), Article ID 1. 